\title{
Vertical current densities and magnetic gradients in sunspots
}

\author{
H. Balthasar
}

Astrophysikalisches Institut Potsdam, An der Sternwarte 16, 14482 Potsdam, Germany

e-mail: hbalthasar@aip.de

Received 14 October 2005 / Accepted 25 November 2005

ABSTRACT

Aims. The vertical component of electric current densities and the height dependence of the vertical component of the magnetic field in sunspots were determined.

Methods. Full-Stokes magnetograms of eight sunspots obtained from infrared spectro-polarimetric measurements were investigated. The magnetic field strength and the magnetic inclination and azimuth were obtained from an inversion code. Vertical current densities and the vertical derivative of the vertical component of the magnetic field strength resulted from Maxwell equations.

Results. It is found that electric current densities and magnetic gradients depend on the finestructure of the sunspots. Typical values for current densities vary in the range $\pm 40 \mathrm{~mA} \mathrm{~m}^{-2}$, but their errors are of the same order. Disturbances in the radial structure of the penumbra are related to enhanced current densities up to $149 \mathrm{~mA} \mathrm{~m}^{-2}$, and a maximum value of $166 \mathrm{~mA} \mathrm{~m}^{-2}$ was found in a light bridge. There are indications that the radial structure of the penumbra is related to current densities, but no correlation to the local intensity fluctuations was found in the outer penumbra. The vertical component of the magnetic field decreases by $0.5-1.5 \mathrm{G} \mathrm{km}^{-1}$ in the umbra. Mean values in the inner penumbra are somewhat smaller than in the umbra, and locally dark structures exhibit a faster decrease with height than bright ones. In the outer penumbra the vertical magnetic component increases, independent of the local intensity distribution.

Conclusions. Electric current densities could be a diagnostic tool for understanding the penumbral finestructure, although the presently available spatial resolution is probably not good enough to avoid artifacts. Magnetic extrapolations might depend crucially on this problem.

Key words. sunspots - Sun: magnetic fields

\section{Introduction}

The solar corona is structured by the magnetic field, but with the present facilities it is fairly difficult to measure the coronal magnetic field directly. Therefore extrapolations of the photospheric magnetic fields are an important approach to understand the coronal structures. A rather simple assumption would be that the magnetic field is a potential field, but most recent methods assume a force-free magnetic field, i.e. electric currents parallel to the magnetic field are possible. Assuming that there is no temporally changing electrical field, the following equation is valid for this case:

$\nabla \times \boldsymbol{B}=\mu \boldsymbol{j}=\alpha \boldsymbol{B}$

where $j$ is the electric current density and $\mu$ the magnetic permeability. If the factor $\alpha$ is constant across the active region, this equation is called linear. Recently, Régnier et al. (2002) and Valori et al. (2005) presented an investigation of the nonlinear case. Generally it is accepted that the magnetic field is force-free in the chromosphere and higher layers, but there are discussions about whether this is also the case in the photosphere. Metcalf et al. (1995) and Georgoulis \& LaBonte (2004) conclude that the photosphere is not force-free, while Moon et al. (2002) claim that the situation is not very far from forcefreeness. Leka \& Metcalf (2003) were not able to reproduce the chromospheric magnetic field doing a force-free extrapolation starting with the photospheric flux.

It is obvious that a good knowledge of the magnetic vector field is crucial for a reliable extrapolation. So far the observations of the magnetic field are either obtained with low spatial resolution or are restricted to small areas like that of a sunspot. Very often only the circular polarization was measured so that the complete magnetic vector remains unknown. It is the aim of the present paper to investigate the magnetic vector field of sunspots and to determine the vertical component of the electric current density with a high spatial resolution, i.e. 1 arcsecond for full Stokes spectro-polarimetry. Electric current sheets play an important role in the models of Schmidt (1991) and Jahn \& Schmidt (1994). Investigations of vertical current densities were previously presented by DeLoach et al. (1984), Ding et al. (1987), and Hagyard (1988) based on vector-magnetographic measurements, as well as by Hofmann et al. (1988) and Hofmann et al. (1989). All these investigations were performed with lower spatial resolution than the present data. The last authors interprete neighboring areas of opposite current direction as a signature of a ring current enclosing an isolated inclined flux bundle.

Electric current densities might also help in the understanding of the fine structure of sunspots. Title et al. (1993) discuss what kind of electric currents should occur in a fluted 
penumbra. Hamedivafa (2003) investigate Joule heating as brightening mechanism for umbral dots, while Hamedivafa \& Sobotka (2004) find indirect observational evidence for this mechanism. Obviously, it is important to search for electric currents in fine structures.

Another important question is why the decrease in the magnetic field strength with height is found to be different depending on the applied method. Mathew et al. (2003) find a value of $4 \mathrm{G} \mathrm{km}^{-1}$ from infrared lines, while Westendorp Plaza et al. (2001) get $1.5-2 \mathrm{G} \mathrm{km}^{-1}$ from lines in the visible. Both groups used different inversion codes. Borrero et al. (2004) obtain a difference of about $800 \mathrm{G}$ over a unit of $\lg \tau$ in the inner penumbra from their $1 \mathrm{C}$-inversion; this difference decreases towards the outer penumbra. Previously, observations of three photospheric lines in the visible yielded 2-3 $\mathrm{G} \mathrm{km}^{-1}$ (Balthasar $\&$ Schmidt 1993). Inversions of a near-infrared group of iron and silicon lines by Sánchez Cuberes et al. (2005) result in $1.7 \mathrm{G} \mathrm{km}^{-1}$ that is fairly independent of position inside the spot. In contrast to this result, Moran et al. (2000) find a gradient of $2 \mathrm{G} \mathrm{km}^{-1}$ near the umbra and about $1 \mathrm{G} \mathrm{km}^{-1}$ in the outer penumbra from a comparison of the magnesium line at $12 \mu \mathrm{m}$ with the iron line at $1564.8 \mathrm{~nm}$. Leka \& Metcalf (2003) come to a similar result when comparing data taken in the $\mathrm{Na} \mathrm{D}_{1}$-line and in the Fe $630 \mathrm{~nm}$ lines. On the other hand, Hofmann \& Rendtel (1989) obtain lower values of about $0.32 \mathrm{G} \mathrm{km}^{-1}$ for the $z$-component of the magnetic field from the condition that the magnetic field is divergence-free.

\section{Observations and data reduction}

The sunspots listed in Table 1 were observed with the Tenerife Infrared Polarimeter (TIP, Martínez Pillet et al. 1997) attached to the spectrograph of the German Vacuum Tower Telescope (VTT), located at the Observatorio del Teide, Tenerife. Measurements of the full Stokes vector were obtained during several observing campaigns from 1999 through 2003 in two different spectral ranges: the regions around Fe I $1564.8 \mathrm{~nm}$ and Fe I $1089.6 \mathrm{~nm}$. The neutral iron line at $1564.8 \mathrm{~nm}$ is often used because of its extremely large Zeeman splitting; the Landé-factor is 3.0, and it is recorded together with the neighboring line at $1565.2 \mathrm{~nm}\left(g_{\mathrm{eff}}=1.45\right)$. The high excitation potentials of these two lines $(5.43 \mathrm{eV}$ and $6.25 \mathrm{eV}$, respectively) make them rather weak in the umbra. With an excitation potential of $3.06 \mathrm{eV}$, the iron line at $1089.6 \mathrm{~nm}$ does not change its strength very much from the quiet sun to the umbra, and there are no indications of blends in the umbra. It exhibits a Zeeman-triplet with a splitting factor of 1.5 , so this line is well-suited for sunspot investigations. For more details of the properties of this line see Balthasar \& Collados (2005).

To obtain the final Stokes-images $I, Q, U$, and $V$, ten sets of single exposures at $50 \mathrm{~ms}$ were added up to increase the signal-to-noise ratio to a value of about 200 in the $Q, U, V$ continuum. Thus the total exposure time was $0.5 \mathrm{~s}$ for each Stokes-parameter. The spots were scanned with a step width of 0.38 . The pixel size corresponded roughly to 0 '. 35 along the slit and depended slightly on the yearly adjustment of the instrument. A full scan took between ten and twenty minutes depending on the number of steps. The image of the huge spot 3 is
Table 1. Observation data. $\theta$ is the heliocentric angle, lat the heliographic latitude, and $\mathrm{cmd}$ is the distance from the central meridian (all angles in degrees). $\lambda$ is the spectral range and $\Delta \lambda$ the spectral pixel size.

\begin{tabular}{lrlrrrrr}
\hline \hline spot & $A R$ date & $\theta$ & lat & cmd & $\lambda[\mathrm{nm}]$ & $\Delta \lambda[\mathrm{pm}]$ \\
\hline 1 & 8693 & $1999-09-13$ & 13.7 & $21 N$ & $1 E$ & 1565.0 & 2.91 \\
2 & 8704 & $1999-09-20$ & 39.6 & $21 S$ & $29 E$ & 1565.0 & 2.91 \\
3 & 9504 & $2001-06-19$ & 8.3 & $8 N$ & $5 E$ & 1089.6 & 3.17 \\
4 & 9516 & $2001-06-30$ & 20.3 & $11 N$ & $18 W$ & 1089.6 & 3.17 \\
5 & 9516 & $2001-07-01$ & 32.9 & $11 N$ & $32 W$ & 1089.6 & 3.17 \\
6 & 9958 & $2002-05-23$ & 23.9 & $4 N$ & $23 W$ & 1089.6 & 2.26 \\
7 & 9970 & $2002-05-28$ & 32.7 & $6 N$ & $32 E$ & 1089.6 & 2.26 \\
8 & 10487 & $2003-10-30$ & 15.0 & $10 N$ & $14 E$ & 1565.0 & 2.99 \\
\hline
\end{tabular}

composed of two scans covering the upper and the lower half, respectively.

The re-composed images of the spots were corrected for geometrical foreshortening and interpolated so that a pixel corresponds to about $250 \mathrm{~km}$ in both spatial directions. The final images are shown in Fig. 1. The spatial resolution achieved in the $1100 \mathrm{~nm}$ range was slightly better than $1 . \prime 0$. The time series obtained for central slit positions of spots 6 and 7 were investigated by Balthasar (2003).

The Stokes parameters were inverted using the SIR-code of Ruiz Cobo \& del Toro Iniesta (1992) in the same way as in Balthasar \& Collados (2005). The code yields a temperature stratification and height independent values for the magnetic field strength, inclination, and azimuth with respect to the line-of-sight (LOS), and the Doppler shift. The lines investigated come from narrow atmospheric layers, so that the height gradients obtained from the inversion would not really be very reliable. The SIR-code also provides error estimates for the derived quantities calculated from the differences in observed and fitted Stokes-profiles.

The azimuthal ambiguity of the magnetic field was resolved by assuming a more or less radial structure inside the spot. Some care is needed to determine the center of azimuth. If needed, the obtained azimuth is changed by $180^{\circ}$ to fulfill this requirement. This procedure worked well for seven spots; a second center of azimuth was needed only for the complex spot 8 , and the two azimuth regimes were divided following the minimum line of the linear polarization. The Cartesian coordinates of the magnetic vector with respect to the LOS were calculated from the total field strength and the magnetic angles, and then this vector is rotated to a frame with respect to the solar surface normal. The final magnetic inclination and azimuth were obtained from these values . Finally, the geometrical foreshortening was removed for the Cartesian components of the magnetic field, and the so obtained values are used in Sects. 3 and 4 . Their errors were obtained by error propagation from the errors calculated by SIR. 


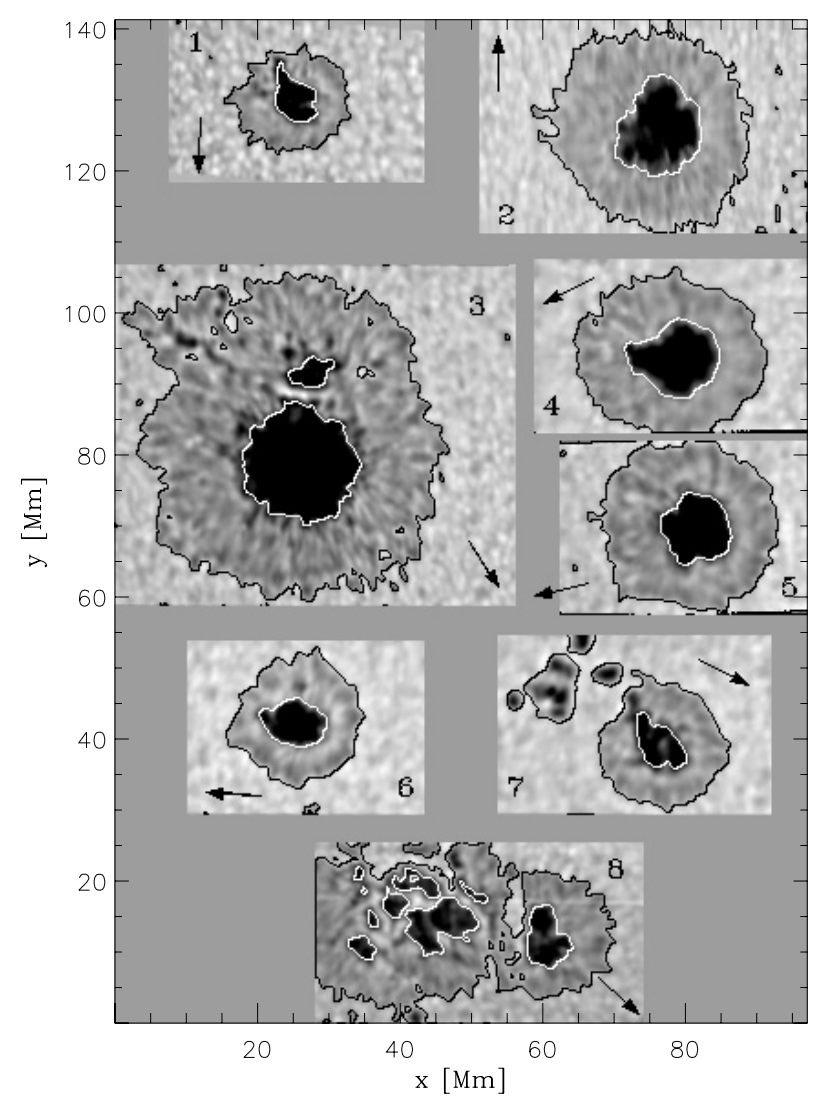

Fig. 1. Infrared intensity maps of the spots, composed of the local continuum near the spectral lines. The intensity contrast is enhanced by an un-sharp masking technique. The numbers refer to the spots in Table 1 . The arrows point towards disk center. Scanning was done from left to right. The penumbral boundaries as used in the following are marked by white and black contours.

\section{Vertical current densities}

The Cartesian components of the magnetic field are displayed in Figs. 2-4. If the integrated absolute circular and linear polarization both drop below a certain value between 0.1 and $0.2 \%$ (depending on the slightly different quality of the data), the remaining polarization signal is regarded to be noise and the corresponding areas are suppressed in the figures. All spots from the northern hemisphere exhibit positive polarity, except that spot 2 from the southern hemisphere has negative polarity. A radial structure in the penumbra is very visible for the vertical magnetic component (see Fig. 2), while fine structures of the horizontal components (see Figs. 3 and 4 are much less related to the radial direction.

Since the magnetic parameters were kept constant during the inversion, I ascribed these values to the layer corresponding to the maximum of the magnetic response function, which was at about -0.5 on the $\lg \tau_{500}$-scale for Fe 1089.6 (Balthasar \& Collados 2005), as well as for Fe 1564.8 (Bellot Rubio et al. 2000). In the geometrical scale of the quiet sun this height corresponds to $70 \mathrm{~km}$. To determine the full vector of the current density, the vertical stratification of the magnetic field must be known. Having only one layer, one can determine the

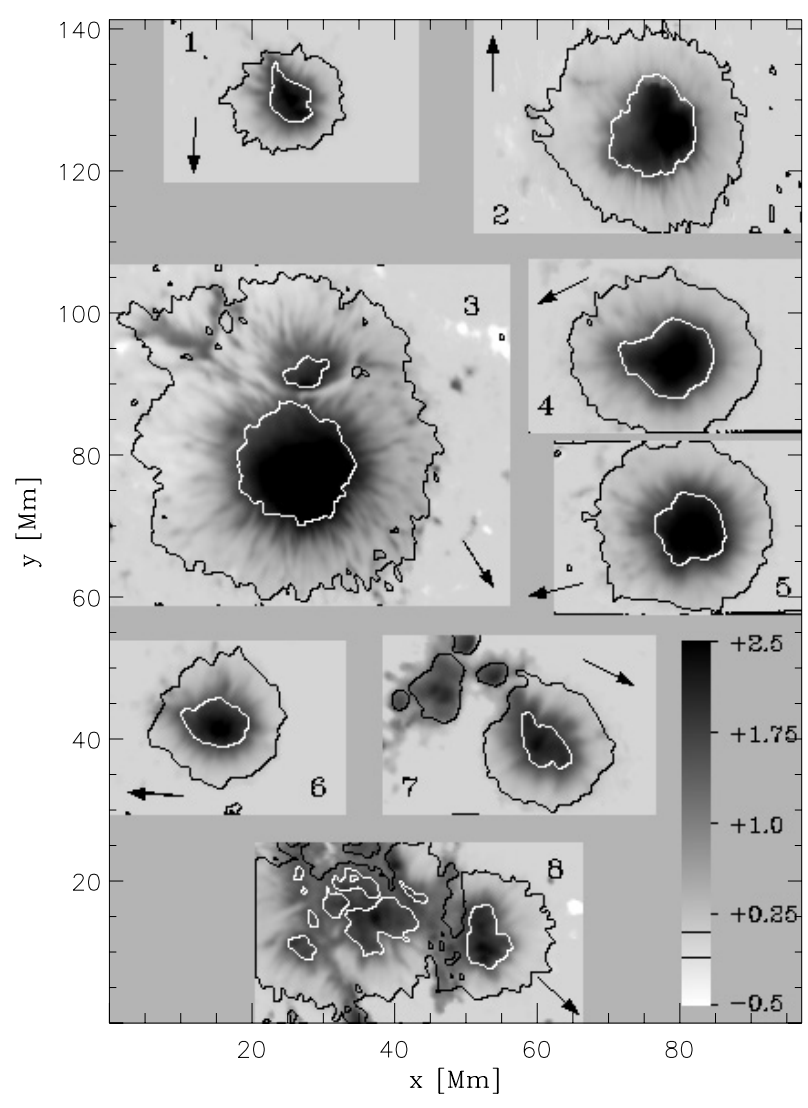

Fig. 2. Vertical component of the magnetic field strength. For the display of spot 2 , the sign has been changed to obtain better visibility of small structures; for this reason, umbral values exceeding $2.5 \mathrm{kG}$ are clipped for all spots. The vertical bar gives the scaling in kilogauss, negative values are clipped at $-0.5 \mathrm{kG}$. The horizontal lines in the scaling bar indicate twice the typical propagated error. The contour lines mark the outer boundaries of umbra and penumbra. Arrows point towards disk center.

horizontal derivatives of the magnetic field that provide the vertical component of $\operatorname{curl} \boldsymbol{B}$ :

$(\nabla \times \boldsymbol{B})_{z}=\frac{\partial B_{y}}{\partial x}-\frac{\partial B_{x}}{\partial y}$.

The partial derivatives were determined from the difference of the two neighboring pixels. This component is proportional to the $z$-component of the electric current density (see equation in the introduction). DeLoach et al. (1984), Hofmann et al. (1988, 1989) applied this method earlier.

The results are shown in Fig. 5 and the corresponding errors in Fig. 6. Typical values inside the sunspots are in the range $\pm 40 \mathrm{~mA} \mathrm{~m}^{-2}$ (selected as limits for Fig. 5). Most values are quite small and are exceeded by their errors. Table 2 contains minimum and maximum values, mean and rms-values, and the mean propagated errors for the penumbrae. Rather high values are obtained at the outer boundary of the penumbra, but these values are quite unrealistic because of the uncertainties of the magnetic field due to low polarization signals. It is obvious that the radial structure of the penumbra prints through to the current densities, although the limited resolution might not allow the current densities to be associated precisely to the bright and dark penumbral filaments. No correlation is detectable from 


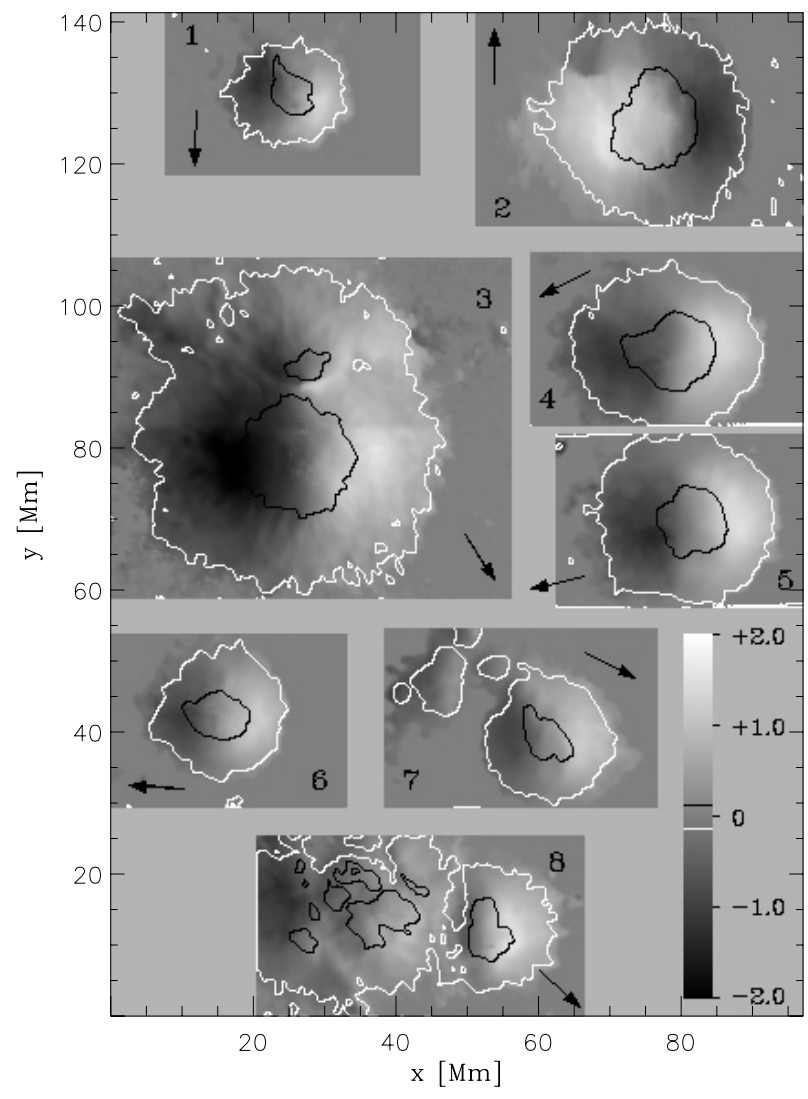

Fig. 3. Horizontal magnetic field component $B_{x}$ perpendicular to the slit. The vertical bar gives the scaling in kilogauss. The horizontal lines in the scaling bar indicate twice the typical propagated error. The contour lines mark the outer boundaries of umbra and penumbra. Arrows point towards disk center.

the present data. Disturbances in the radial structure of the penumbra show up as enhanced current densities. A maximum of $149 \pm 44 \mathrm{~mA} \mathrm{~m}^{-2}$ is found for that of spot 2 in the upper left, and neighboring pixels vary around $100 \pm 45 \mathrm{~mA} \mathrm{~m}^{-2}$. The most pronounced feature of spot 3 occurs in the range between the two umbrae; positive and negative current directions are close together. The extreme values are $+166 \pm 96 \mathrm{~mA} \mathrm{~m}^{-2}$ and $-100 \pm 62 \mathrm{~mA} \mathrm{~m}^{-2}$.

In the umbra small, more circular structures are present. However, the intensity is low here and the accuracy of the polarization signals is less than for the penumbra, especially for spots 1, 2, and 8 that are observed in the $1565 \mathrm{~nm}$ lines, which become rather weak in the umbra. In addition, the horizontal components of the magnetic field, which are responsible for the vertical current densities, are small compared to the vertical magnetic component, and their errors are high. In spot 6 there is a ring of current densities inside the umbra. Spots 4 and 5 exhibit an extended area of negative current densities across the umbra-penumbra transition on the right side. Possibly these are indications of the peripatopause introduced in the models of Schmidt (1991) and Jahn \& Schmidt (1994).

Comparing Figs. 2 and 5, it is clear that the parameter $\alpha$ of the force-free case changes its sign across the sunspot very frequently, and its amount varies by several orders of magnitude.

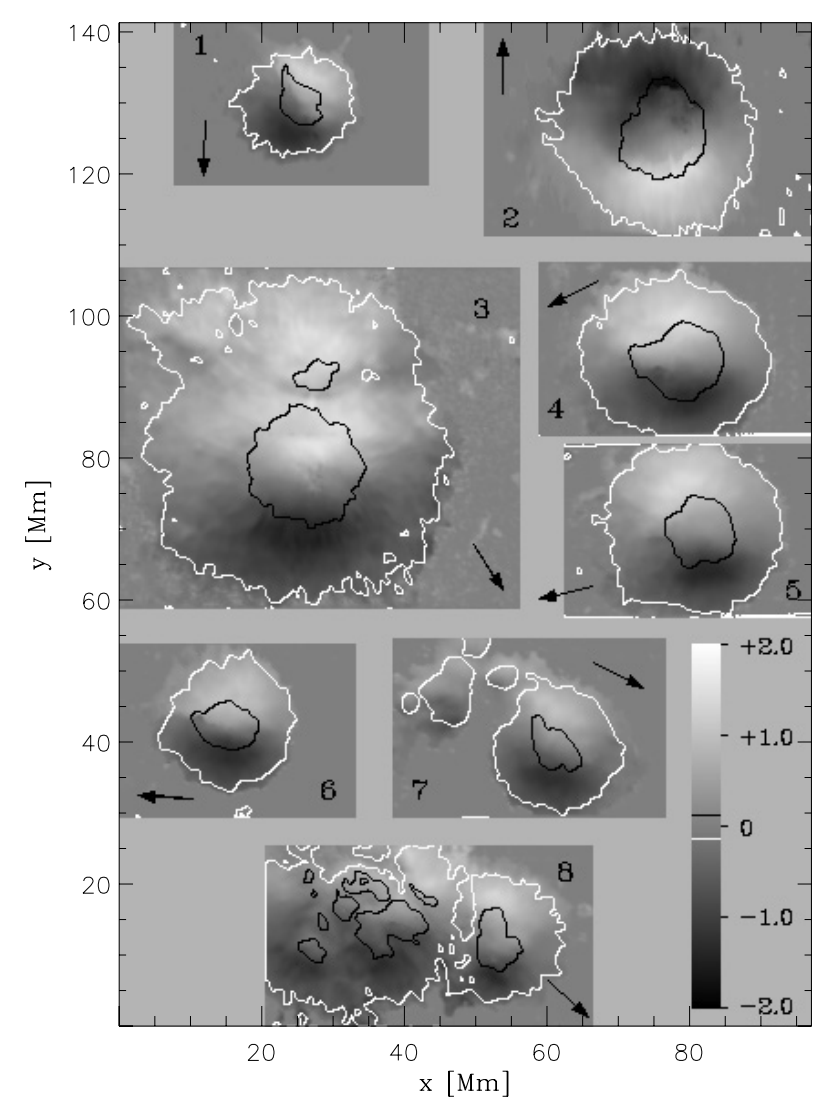

Fig. 4. Horizontal magnetic field component $B_{y}$ parallel to the slit. The vertical bar gives the scaling in kilogauss. The horizontal lines in the scaling bar indicate twice the typical propagated error. The contour lines mark the outer boundaries of umbra and penumbra. Arrows point towards disk center.

\section{The height dependence of the magnetic z-component}

Using $\operatorname{div} \boldsymbol{B}=0$, one can determine the vertical dependence of the vertical component of the magnetic field in a similar technique:

$\frac{\partial B_{z}}{\partial z}=-\left(\frac{\partial B_{x}}{\partial x}+\frac{\partial B_{y}}{\partial y}\right)$

The same method was applied by Hofmann \& Rendtel (1989).

In Fig. 7 the results for the vertical derivatives of the vertical magnetic component are displayed, and the corresponding errors are in Fig. 8. Table 3 gives minimum and maximum, mean and rms values over the regarded area, together with the mean propagated error. Those parts of the penumbra are considered as inner penumbra where the vertical component of the magnetic field exceeds $1000 \mathrm{G}$, which is the value that occurs in the one-component inversion at the location of the sharp transition from a steep to a more horizontal magnetic field in one component of the two-component inversion of Bellot Rubio et al. (2004). In the umbra and inner penumbra, a decrease with height is usually encountered. There is a clear tendency in the inner penumbra for the vertical magnetic component to decrease faster with height in locally dark areas. In the outer penumbra, no dependence on the intensity fine structure can be detected. This behavior is demonstrated in Figs. 9 and 10 . 


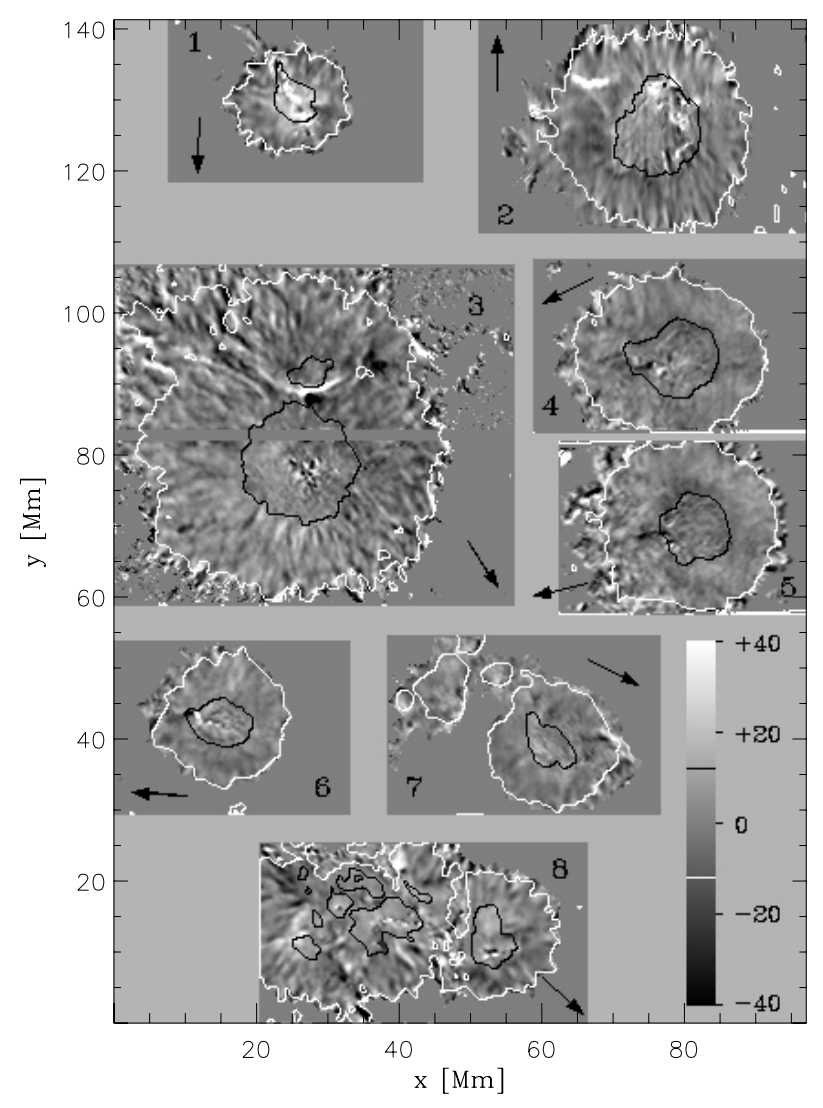

Fig. 5. Vertical current densities for the eight spots. Absolute values of more than $40 \mathrm{~mA} \mathrm{~m}^{-2}$ are cut to enhance the visibility of small scale variations. The scaling is given by the vertical bar, while horizontal lines in the bar indicate the typical rms-range. The black line indicates the umbral boundary, and the white line the border between penumbra and quiet sun. The arrows point towards disk center. Note that the image of spot 3 is combined from two scans, therefore a horizontal strip in the middle of the image is not reliable because of the time difference.

The intensity variations used for these figures are obtained by subtracting a smoothed image from the original intensities. In this case, the full width of the smoothing window was 15 pixel (corresponding to $3750 \mathrm{~km}$ on the Sun) in both directions.

In the umbra, typical values are around $-0.5 \mathrm{G} \mathrm{km}^{-1}$, but in some locations the decrease exceeds $-1.0 \mathrm{G} \mathrm{km}^{-1}$. These values are higher than the $0.3-0.4 \mathrm{G} \mathrm{km}^{-1}$ obtained by Hofmann $\&$ Rendtel (1989). In the outer penumbra and outside the spot, the vertical field strength increases with height, in agreement with Hofmann \& Rendtel (1989). The extreme value for spot 3 is $1.7 \pm 0.3 \mathrm{G} \mathrm{km}^{-1}$. This can be explained if the magnetic field is less inclined in higher layers (increase of the z-component only) as reported by Westendorp Plaza et al. (2001), but Borrero et al. (2004) find an increase of the inclination with height, while Sánchez Cuberes et al. (2005) claim that the inclination is independent of height. The $z$-component of the magnetic field would also increase with height in the case of a return flux as predicted in the model of Osherovic \& Garcia (1989) and as found in inversions with two atmospheric components for one of them, see del Toro et al. (2001), Bellot Rubio et al. (2004), Borrero et al. (2004), Sánchez Almeida (2005),

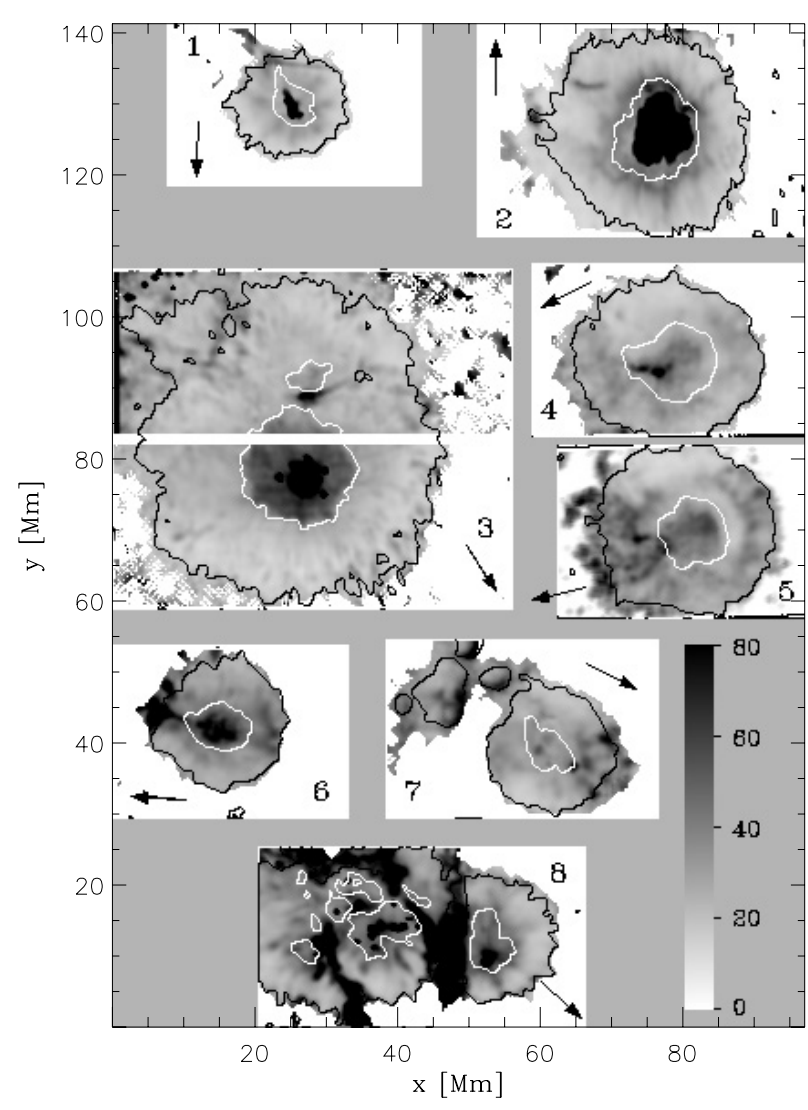

Fig. 6. Propagated errors of the vertical current densities for the eight spots. Values of more than $80 \mathrm{~mA} \mathrm{~m}^{-2}$ are cut. The scaling is given by the vertical bar. The white line indicates the umbral boundary, and the black line the border between penumbra and quiet sun. The arrows point towards disk center.

Table 2. Minimum, maximum, mean, rms-values, and mean errors of the individual values for the vertical current densities in the penumbrae of the different spots with values given in $\mathrm{mA} \mathrm{m}^{-2}$.

\begin{tabular}{llrrrrrr}
\hline \hline spot date & \multicolumn{2}{l}{$\min$} & $\max$ & mean & rms & err \\
\hline 1 & $1999-09-13$ & -59 & 54 & +0.9 & 12 & 24 \\
2 & $1999-09-20$ & -114 & 149 & +0.1 & 14 & 24 \\
3 & $2001-06-19$ & -100 & 166 & -1.2 & 13 & 23 \\
4 & $2001-06-30$ & -38 & 48 & -0.3 & 6 & 25 \\
5 & $2001-07-01$ & -57 & 66 & +0.5 & 9 & 32 \\
6 & $2002-05-23$ & -38 & 25 & +0.4 & 6 & 36 \\
7 & $2002-05-28$ & -62 & 41 & -0.1 & 8 & 34 \\
8 & $2003-10-30$ & -101 & 66 & +0.1 & 12 & 51 \\
\hline
\end{tabular}

and Langhans et al. (2005). The present one-component inversion yields an inverse sign of $B_{z}$ only for spot 3 on the left side (hardly visible in the scale of Fig. 2). A magnetic canopy would cause an increase in the total field strength with height.

An increase of the $B_{z}$ is also observed in the disturbed area between the two umbrae of spot 3 , where enhanced current densities are encountered; here the maximum values is $0.8 \pm 0.5 \mathrm{G} \mathrm{km}^{-1}$. A relation to the fine structure is not as obvious as for the current densities, but small scale changes (even of the sign) are present. 


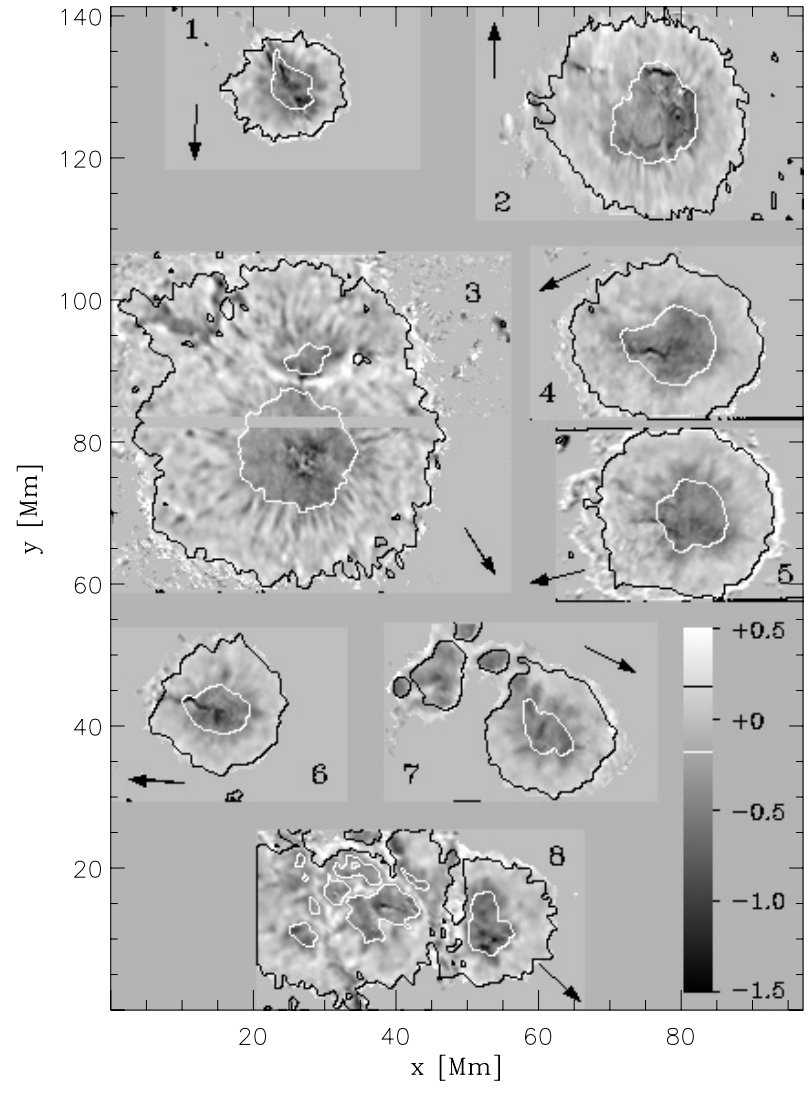

Fig. 7. Vertical derivative of the vertical component of the magnetic field strength for the eight spots. The sub-image of spot 2 (negative polarity) is displayed sign-reversed. Values of more than $+0.5 \mathrm{G} \mathrm{km}^{-1}$ and less than $-1.5 \mathrm{G} \mathrm{km}^{-1}$ are cut to enhance the visibility of small scale variations. The scaling is given by the vertical bar. The contour lines indicate the outer boundaries of umbra and penumbra. The arrows point towards disk center. Note that the image of spot 3 is combined from two scans, so a horizontal strip in the middle of the image is not reliable. The horizontal lines in the scaling bar indicate the typical rms-range.

\section{Discussion and conclusions}

The results presented in this paper depend on two crucial conditions: The neighboring magnetic components must belong to the same height layer, and they must belong to the same magnetic finestructure. Sütterlin (2001) and Balthasar et al. (2001) found a typical width of $250 \mathrm{~km}$ for penumbral filaments, and now there are results from the new Swedish solar telescope that even smaller structures are present (see Rouppe van der Voort et al. 2004). Bellot Rubio et al. (2003, 2004) demonstrate that the penumbra consists of at least two unresolved magnetic components, which stem not necessarily from the same geometrical height. If the neighboring values used to determine the partial derivatives belong to different finestructures, the obtained values become meaningless. Problems also occur where the Wilson depression changes strongly from pixel to pixel.

Error estimates of SIR contain systematic errors, as well as pure noise. Differences in the magnetic field components between neighboring pixel might be more reliable than the absolute values because the systematic influences would act in the same direction for all pixel in a certain area. This argument is

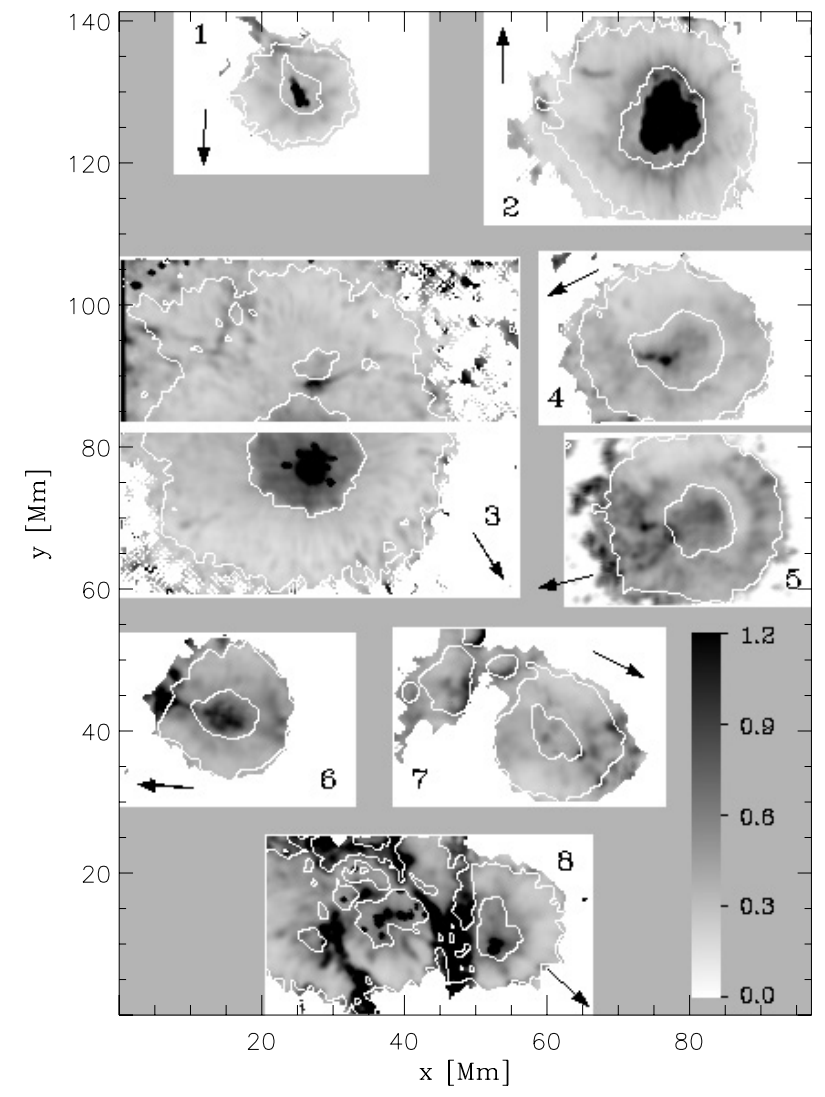

Fig. 8. Errors of the vertical derivative of the vertical component of the magnetic field strength for the eight spots. Values are cut at $1.2 \mathrm{G} \mathrm{km}^{-1}$. The scaling is given by the vertical bar. The contour lines indicate the outer boundaries of umbra and penumbra. The arrows point towards disk center.

Table 3. Minimum, maximum, mean, rms-values, and mean errors of the individual values for the vertical gradient of the magnetic $z$ component $\left(B_{z}\right)$ of the different spots with values given in $\mathrm{G} \mathrm{km}^{-1}$. The upper half is for the inner penumbra, the lower for the outer penumbra.

\begin{tabular}{llllllll}
\hline \hline spot & date & min & max & mean & rms & err \\
\hline 1 & $1999-09-13$ & -1.4 & 0.0 & -0.44 & 0.19 & 0.38 \\
2 & $1999-09-20$ & -1.3 & 0.2 & -0.27 & 0.18 & 0.52 \\
3 & $2001-06-19$ & -1.4 & 0.4 & -0.21 & 0.22 & 0.33 \\
4 & $2001-06-30$ & -0.7 & 0.1 & -0.23 & 0.13 & 0.35 \\
5 & $2001-07-01$ & -1.0 & 0.2 & -0.26 & 0.17 & 0.40 \\
6 & $2002-05-23$ & -1.0 & 0.1 & -0.27 & 0.14 & 0.52 \\
7 & $2002-05-28$ & -0.9 & 0.6 & -0.30 & 0.19 & 0.47 \\
8 & $2003-10-30$ & -1.1 & 1.0 & -0.18 & 0.26 & 0.94 \\
\hline 1 & $1999-09-13$ & -0.7 & 0.6 & +0.06 & 0.17 & 0.26 \\
2 & $1999-09-20$ & -1.1 & 2.4 & +0.06 & 0.17 & 0.28 \\
3 & $2001-06-19$ & -1.2 & 1.7 & +0.05 & 0.18 & 0.27 \\
4 & $2001-06-30$ & -0.5 & 0.7 & +0.04 & 0.11 & 0.31 \\
5 & $2001-07-01$ & -0.6 & 1.1 & +0.07 & 0.14 & 0.39 \\
6 & $2002-05-23$ & -0.6 & 0.4 & +0.05 & 0.11 & 0.43 \\
7 & $2002-05-28$ & -0.4 & 0.8 & +0.04 & 0.11 & 0.38 \\
8 & $2003-10-30$ & -0.6 & 0.7 & +0.05 & 0.14 & 0.41 \\
\hline
\end{tabular}



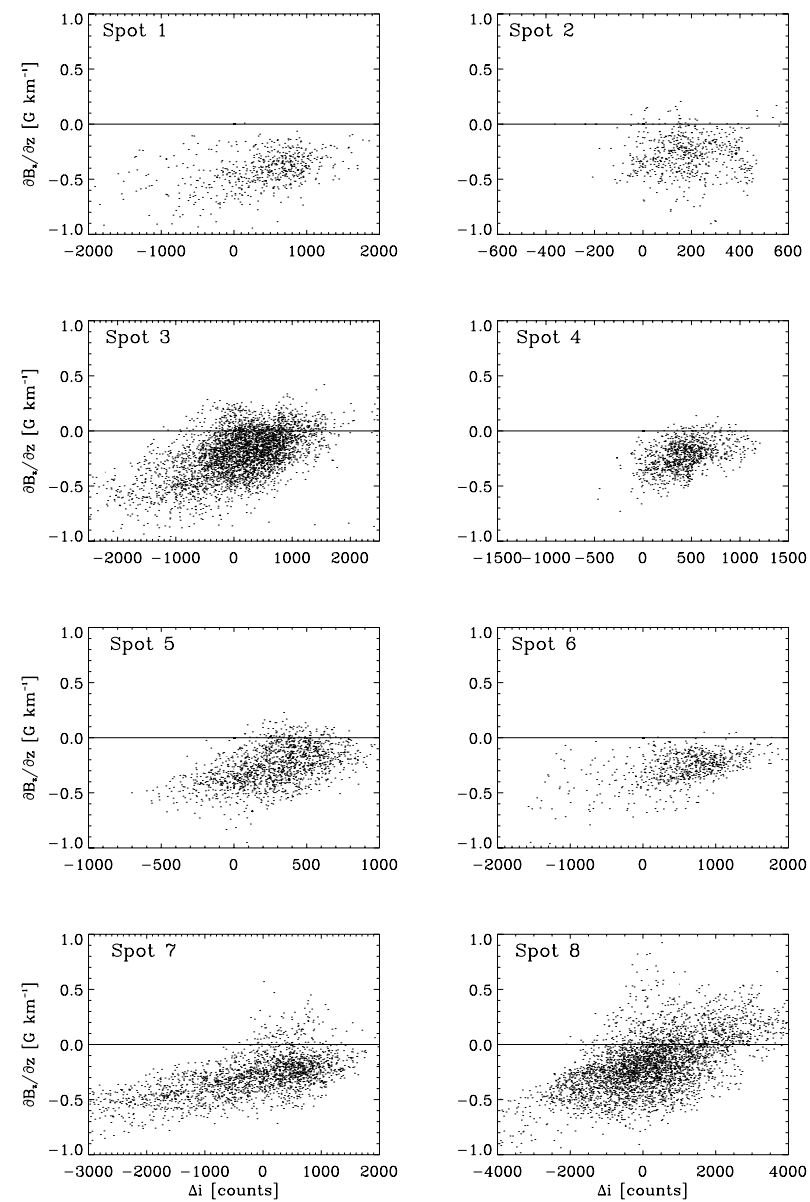

Fig. 9. Scatter plots of the vertical derivative of the vertical component of the magnetic field strength versus local intensity fluctuations for the inner penumbrae.

supported by the fact that the rms-values given in Tables 2 and 3 are clearly smaller than the mean propagated errors. However, the errors are treated as noise in the error propagation.

The main conclusions can be summarized as follows:

1. The resolution of the finestructure is important for the investigation of electric current densities. The present data indicate a relation to the penumbral finestructure, but a correlation to intensity structures could not be established.

2. Disturbances of the radial structure of the penumbra as in the upper left of spot 2 are related to enhanced current densities up to $149 \mathrm{~mA} \mathrm{~m}^{-2}$, and in a light bridge even $166 \mathrm{~mA} \mathrm{~m}^{-2}$ are observed.

3. With the present spatial resolution, the magnetic $z$-component $\left(B_{z}\right)$ decreases faster with height in the umbra and inner penumbra than for previous observations with lower resolution. This decrease is faster in locally dark structures than in bright ones in the inner penumbra.

4. In the outer penumbra, an increase in the magnetic $z$-component $\left(B_{z}\right)$ is found, independent of the local intensity structure.

If the opposite current densities in the penumbrae in Fig. 5 are real, i.e. they are not produced by unresolved finestructures, they could be interpreted in a similar way as in
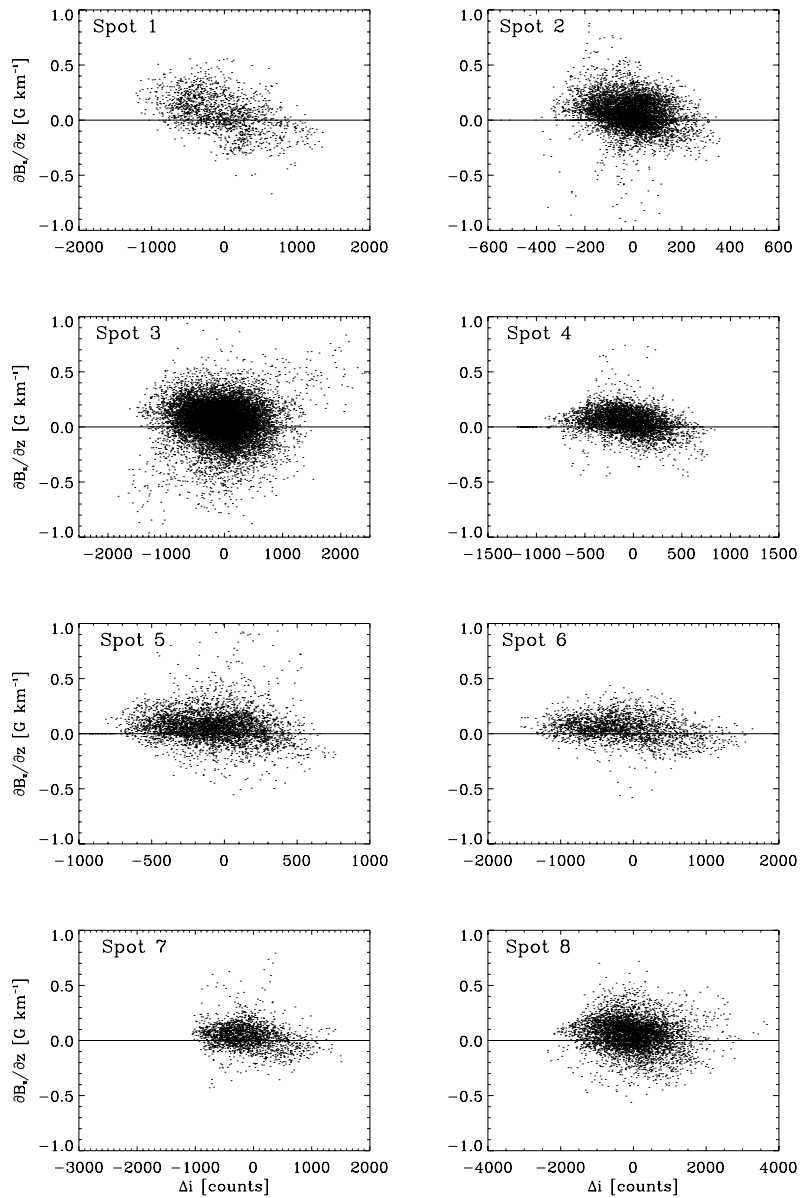

Fig. 10. Scatter plots of the vertical derivative of the vertical component of the magnetic field strength versus local intensity fluctuations for the outer penumbrae.

Hofmann et al. (1988, 1989): these could be indications of ring currents around inclined fluxtubes forming the penumbra.

Results inside the umbra, especially the small scale variations, are surely affected by the low accuracy of the polarization signals (because of low intensity, and also for Fe $1565 \mathrm{~nm}$ because of the weakness of the lines) and by insufficient spatial resolution. The spatial resolution of the present data is not sufficient for identifying individual umbral dots (see Socas Navarro et al. 2004).

The increase in the magnetic $z$-component in the outer penumbra can be explained if the field lines are almost horizontal in deep layers and less inclined in higher layers. Such a behavior of the magnetic field would be observed especially in the case of return flux, as in the model of Osherovic \& Garcia (1989). Close to the outer boundary of the penumbra, another possible explanation would be that the magnetic field forms a canopy above a non-magnetic layer.

For a more sophisticated investigation, a much higher spatial resolution is required as will be provided by a new generation of telescopes like GREGOR (see Volkmer et al. 2005) or the ATST (see Keil et al. 2004). Several spectral lines sensitive to different heights should be observed simultaneously. Then it becomes possible to determine all partial derivatives, which are 
needed to determine the full vector of current densities or how strong the Lorentz-forces are.

Acknowledgements. I am indebted to A. Hofmann for his suggestions for improving the paper. I wish to thank my colleagues from the Instituto de Astrofísica de Canarias for the possibility to use TIP and the SIR-inversion code. The Vacuum Tower Telescope in Tenerife is operated by the Kiepenheuer-Institut für Sonnenphysik (Germany) in the Spanish Observatorio del Teide of the Instituto de Astrofísica de Canarias.

\section{References}

Balthasar, H. 2003, Sol. Phys., 218, 85

Balthasar, H., \& Collados, M. 2005, A\&A, 429, 705

Balthasar, H., \& Schmidt, W. 1993, A\&A, 279, 243

Balthasar, H., Sütterlin, P., \& Collados, M. 2001, Astron. Nachr., 322, 367

Bellot Rubio, L. R., Balthasar, H., \& Collados, M. 2004, A\&A, 427, 319

Bellot Rubio, L. R., Balthasar, H., Collados, M., \& Schlichenmaier, R. 2003, A\&A, 403, L47

Bellot Rubio, L. R., Collados, M., Ruiz Cobo, B., \& Rodríguez Hidalgo, I. 2000, ApJ, 543, 989

Borrero, J. M., Solanki, S. K., Bellot Rubio, L. R., Lagg, A., \& Mathew, S. K. 2004, A\&A, 422, 1093

DeLoach, A. C., Hagyard, M. J., Rabin, D., et al. 1984, Sol. Phys., 91, 235

Ding, Y. J., Hagyard, M. J., DeLoach, A. C., Hong, Q. F., \& Liu, X. P. 1987, Sol. Phys., 109, 307

Georgoulis, M. K., \& LaBonte, B. J. 2004, ApJ, 615, 1029

Hagyard, M. J. 1988, Sol. Phys., 115, 107

Hamedivafa, H. 2003, A\&A, 407, 761

Hamedivafa, H., \& Sobotka, M. 2004, A\&A, 428, 215

Hofmann, A., \& Rendtel, J. 1989, Astron. Nachr., 310, 61

Hofmann, A., Grigorjev, V. M., \& Selivanov, V. L. 1988, Astron. Nachr., 309, 373

Hofmann, A., Ruždjak, V., \& Vršnak, B. 1989, Hvar Obs. Bull., 13, 11
Jahn, K., \& Schmidt, H. U. 1994, A\&A, 290, 295

Keil, S., Oschmann, J., Rimmele, T. R., et al. 2004, Proc. SPIE Conf., 5489,625

Langhans, K., Scharmer, G. B., Kiselman, D., Löfdal, M. G., \& Berger, T. E. 2005, A\&A, 436, 1087

Leka, K. D., \& Metcalf, T. R. 2003, Sol. Phys., 212, 361

Mathew, S. K., Lagg, A., Solanki, S. K., et al. 2003, A\&A, 410, 695

Martínez Pillet, V., Collados, M., Sánchez Almeida, et al. 1999, in High resolution Solar Physics: Theory, Observations and Techniques, ed. T. R. Rimmele, K. S. Balasubramaniam, \& R. R. Radick, ASP Conf. Ser., 183, 264

Metcalf, T. R., Jiao, L., McClymont, A. N., Canfield, R. C., \& Uitenbroek, H. 1995, ApJ, 439, 474

Moon, Y. J., Choe, G. S., Yun, H. S., Park, Y. D., \& Mickey, D. L. 2002, ApJ, 568, 422

Moran, T., Deming, D., Jennings, D. E., \& McCabe, G. 2000, ApJ, 533,1035

Osherovic, V. A., \& Garcia, H. A. 1989, ApJ, 336, 468

Régnier, S., Amari, T., \& Kersale, E. 2002, A\&A, 392, 1119

Rouppe van der Voort, L. H. M., Löfdahl, M. G., Kiselman, D., \& Scharmer, G. B. 2004, A\&A, 414, 717

Ruiz Cobo, B., \& del Toro Iniesta, J. C. 1992, ApJ, 398, 375

Sánchez Almeida, J. 2005, ApJ, 622, 1292

Sánchez Cuberes, M., Puschmann, K. G., \& Wiehr, E. 2005, A\&A, 440,345

Schmidt, H. U. 1991, Geophys. Astrophys. Fluid Dyn., 62, 249

Socas Navarro, H., Martínez Pillet, V., Sobotka, M., \& Vázquez, M. 2004, ApJ, 614, 448

Sütterlin, P. 2001, A\&A, 374, L21

Title, A. M., Frank, Z. A., Shine, R. A., et al. 1993, ApJ, 403, 780

del Toro Iniesta, J. C., Bellot Rubio, L. R., \& Collados, M. 2001, ApJ, 549, L139

Valori, G., Kliem, B., \& Keppens, R. 2005, A\&A, 433, 335

Volkmer, R., von der Lühe, O., Kneer, F., et al. 2005, Proc. SPIE Conf., 5489, 693

Westendorp Plaza, C., del Toro Iniesta, J. C., Ruiz Cobo, B., et al. 2001, ApJ, 547, 1130 